\title{
Análisis del Comportamiento del tráfico en Internet durante la Pandemia del Covid-19: el caso de Colombia ${ }^{1}$
}

\section{Analysis of Internet Traffic Behavior during the Covid-19 Pandemic: the case of Colombia}

\author{
J. J. Padilla \\ Recibido: junio 1 de 2020 - Aceptado: noviembre 23 de 2020.
}

\begin{abstract}
Resumen-Una de las situaciones más críticas en todo el mundo en el año 2020 es la pandemia del Covid-19. Todas las áreas de la sociedad han colapsado debido a la cuarentena declarada en diferentes países. Uno de los recursos de infraestructura más importantes en esta situación ha sido el servicio de Internet debido a que las personas deben trabajar o estudiar desde sus casas, y tales conexiones se realizan por medio de Internet. Por tanto, los proveedores de servicio de Internet (ISPs) han tenido que soportar este tráfico, considerado atípico hasta ese momento. En este artículo se realiza un análisis del comportamiento del tráfico de Internet sobre los ISPs de Colombia para comprender qué ocurrió en estas redes durante esta situación en los meses de marzo y abril de 2020. Además, se realiza un estimativo del incremento necesario en los recursos de las redes de los ISPs, para lo que se utilizó un modelo de red de cola cerrada (CQN). Los resultados muestran que el tráfico se incrementó en la mayoría de los operadores en una media del $29.76 \%$ en marzo y una media del $\mathbf{5 1 . 5 4 \%}$ en abril de 2020. Los recursos, por su parte se debieron incrementar en un $28.3 \%$ en marzo y un $32.71 \%$ en abril.
\end{abstract}

Palabras clave-Covid-19, pandemia, teletráfico, modelo de desempeño, análisis de tráfico.

\begin{abstract}
One of the most critical situations in all world in 2020 year is the Covid-19 pandemic. All areas of society have colapsed due to the quarantine declared in several countries. One of the most important infrastructure resources in this situation is the Internet service because people have to work and study from home and such people connections have performed by Internet. Thus, Internet Service Providers had to endure increased traffic. In this paper, an analysis of Internet traffic over ISPs at
\end{abstract}

\footnotetext{
${ }^{1}$ Producto apoyado por la Universidad Pontificia Bolivariana través de la Facultad de Ingeniería Eléctrica y Electrónica.

J. J. Padilla, Universidad Pontificia Bolivariana, Bucaramanga, Colombia, email: jhon.padilla@upb.edu.co.
}

Como citar este artículo: Padilla, J. J. Análisis del Comportamiento del tráfico en Internet durante la Pandemia del Covid-19: el caso de Colombia, Entre Ciencia e Ingeniería, vol. 14, no. 28, pp. 26-33, julio - diciembre 2020. DOI: https://doi.org/ 10.31908/19098367.2012.

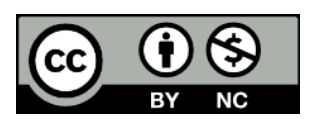

Attribution-NonCommercial 4.0 Intenational (CC By-NC 4.0)
Colombia was performed to understand what happened over such networks. Also, an estimate was made of the increase in network resources by means of a Closed Queued Network model. Results shows that Internet traffic increased for almost all operators with a percentage mean of 29.76 in March and 51.54 in April, and besides, network resources increased in a percentage near to 28.3 in March and 32.71 in April.

Keywords-Covid-19, pandemic, teletraffic, internet, performance model, traffic analysis.

\section{INTRODUCCIÓN}

$\mathrm{U}$ NO de los mayores problemas que ha tenido nuestra sociedad en los últimos tiempos es el de la pandemia debida al virus COVID-19. Esta pandemia ha puesto a los gobiernos de los diferentes países del mundo a tomar medidas rigurosas tales como la cuarentena, que ha consistido en que la gran mayoría de personas deben permanecer en sus casas por un gran período de tiempo, esto con el fin de evitar los contagios debido a que cierto porcentaje de la población muere por este virus. Al permanecer confinados la mayoría de habitantes, las telecomunicaciones se han vuelto una herramienta indispensable para seguir trabajando desde casa en muchos casos. También se han creado nuevas maneras de relacionarnos a través de las redes, al punto de por ejemplo llegar a hacer celebraciones familiares y reuniones de amigos por teleconferencia, algo que antes solo se dejaba para situaciones muy especiales, ahora se convirtió en algo común y que pasó a ser parte de nuestra manera de vivir. Pero al ocurrir estos cambios, las redes de comunicaciones que soportan el Internet han tenido que soportar más tráfico y nuevos servicios que antes no existían o no eran tan comunes. Las empresas tanto privadas como del estado, han tenido que volcar sus innovaciones hacia la manera de permitir que se hagan muchas de las transacciones o diligencias por internet, desde pagos, hasta suscripciones y solicitudes varias. Los colegios y universidades han tenido que migrar hacia plataformas para dar clases a través de Internet mediante encuentros por teleconferencias, videos, plataformas de elearning, etc. Internet se ha vuelto indispensable en esta situación de pandemia. Esto ha traído un mayor uso de las 
redes de telecomunicaciones $\mathrm{y}$, por tanto, un aumento de tráfico. Se han hecho algunos esfuerzos por estudiar estos cambios en las redes, pero en su mayoría aparecen en páginas web especializadas de ciertas empresas de consultoría, más no hay mucha literatura científica con respecto al efecto de la pandemia en las redes de telecomunicaciones e internet. Por tanto, este artículo pretende hacer un estudio del efecto en el tráfico de las redes debido a la pandemia por el covid-19 específicamente en Colombia. Este estudio se basó en los datos entregados por la CRC (Comisión de Regulación de Comunicaciones) en sus informes sobre el tráfico de los diferentes operadores de internet en el país [1]. Se tomaron como base los datos de los meses de febrero a abril de 2020.

\section{ESTADO DEL ARTE}

Sobre el efecto de la pandemia en el tráfico de internet, se han realizado algunos análisis preliminares que se han descrito en diferentes páginas web especializadas y en algunos artículos que describiremos brevemente a continuación.

En [2], los autores hicieron un análisis del tráfico en la red de la universidad Politécnico de Milán y cómo la pandemia aumentó dicho tráfico y pudieron comprobar que la red pudo ser lo suficientemente robusta para soportarlo.

En [3], la autora hizo un análisis de diferentes países en el mundo desarrollado y concluye que el tráfico de internet en estos países aumentó en promedio en un $30 \%$ con respecto al tráfico normal del mes de Marzo en años anteriores. También menciona que los ataques de denegación de servicio aumentaron en el operador Akamai. Además, describe que en Europa el tráfico debido a las video conferencias y a la navegación web aumentó considerablemente con respecto a otros servicios como video juegos, servicios peer to peer y social media.

Por su parte, en [4] los autores hicieron un análisis del aumento de tráfico en internet en diferentes países desarrollados y la disminución en la velocidad de descarga de los usuarios como una medida de la afectación en la calidad de la experiencia. Se puede ver en la Tabla I un resumen de estos parámetros para diferentes países según aparece en dicho estudio.

TABLA I

CAMBIOS EN EL TRÁFICO Y VELOCIDAD DE DESCARGA PARA DIFERENTES PAÍSES [4]

\begin{tabular}{|l|l|l|}
\hline País o Estado & Cambio tráfico & Cambio velocidad \\
\hline Francia & +38.4 & -13.9 \\
\hline Italia & +109.3 & -35.4 \\
\hline Japón & +31.5 & -9.7 \\
\hline Reino Unido & +78.6 & -30.3 \\
\hline California-USA & +46.5 & -1.2 \\
\hline Michigan-USA & +37.9 & -16.1 \\
\hline New York y New Jersey & +44.6 & -5.5 \\
USA & & \\
\hline
\end{tabular}

Otro análisis interesante en este documento fue el tipo de servicios que ocuparon el tráfico y su aumento en tráfico durante la cuarentena. Así, los servicios de streaming aumentaron un porcentaje de 29.6, los servicios de publicidad digital aumentaron un porcentaje de 70.6 , por su parte las redes sociales aumentaron un $40.88 \%$, los memes y los gif un $30.28 \%$, los video juegos un $28.54 \%$ y los servicios de educación un $34.55 \%$.
En [5] encontramos un documento en el cual se tomaron datos de una compañía denominada Kaspr Datahaus, la cual midió el porcentaje de cambio en el RTT (Rount Trip Time ó Tiempo de ida y vuelta) de los paquetes al ir desde estados unidos hacia diferentes regiones durante un período normal y durante el período de cuarentena en diferentes países y a este cambio de velocidad le denominó Presión de Internet. Estos datos se obtuvieron para diferentes países del mundo que tuviesen al menos 100 infectados por covid-19 y estuvieran en medidas especiales de aislamiento. Según el gráfico que muestran, los países con peores resultados son Malasia (6\%), Suiza $(10 \%-12 \%)$ e Irán $(25 \%)$.

\section{ANÁLISIS DE LOS CAMBIOS DE TRÁFICO EN COLOMBIA}

Para Colombia tenemos dos conjuntos de datos, el primero es el de la empresa Kaspr Datahaus sobre la presión de Internet para Colombia [5], y otro conjunto de datos dados por la CRC (Comisión de Regulación de Comunicaciones) del gobierno de Colombia sobre datos de tráfico en los diferentes operadores de Telecomunicaciones [1]. Por tanto, iniciaremos nuestro estudio con los datos de Kaspr Datahaus y luego haremos el análisis de los datos de la CRC.

\section{A. Presión de Internet en Colombia}

Si bien Colombia no aparece en el análisis realizado en [4], en la página de Kaspr Datahaus sí se encuentran los datos de nuestro país. Por tanto, aquí se presenta la tabla II con la presión de internet para diferentes regiones de Colombia.

\section{TABLA II}

CAMBIOS EN EL TRÁFICO Y VELOCIDAD DE DESCARGA PARA DIFERENTES REGIONES DE COLOMBIA

\begin{tabular}{|c|c|}
\hline Región & Presión de Internet \\
\hline Cundinamarca & 0 \\
\hline Huila & 9.98 \\
\hline Magdalena & 19.03 \\
\hline Nariño & 12.64 \\
\hline Norte de Santander & 6.12 \\
\hline Qundío & 12 \\
\hline Risaralda & 7.55 \\
\hline Santander & 5.41 \\
\hline Tolima & 12.29 \\
\hline Valle del Cauca & 4.46 \\
\hline Atlántico & 9.68 \\
\hline Bolívar & 10.25 \\
\hline
\end{tabular}

Se observa en la Tabla II que hubo medidas de presión de Internet muy deficientes para Colombia si comparamos con los países que se mencionó en el documento como Suiza e Irán. El valor máximo fue de $19.03 \%$ para el caso de Magdalena y valores también deficientes (entre $8 \%$ y 13\%) en Tolima, Quindío, Nariño, Bolívar, Atlántico y Huila. Los Departamentos con valores más aceptables (entre 4\% y $8 \%$ ) fueron Valle del Cauca, Santander, Risaralda y Norte de Santander. Curiosamente, la región de Cundinamarca aparece con $0 \%$, lo cual podría deberse a que allí se encuentran los centros principales de los diferentes operadores de Internet. En general, el valor medio para Colombia fue de $9.11 \%$, lo cual no es nada favorable. 


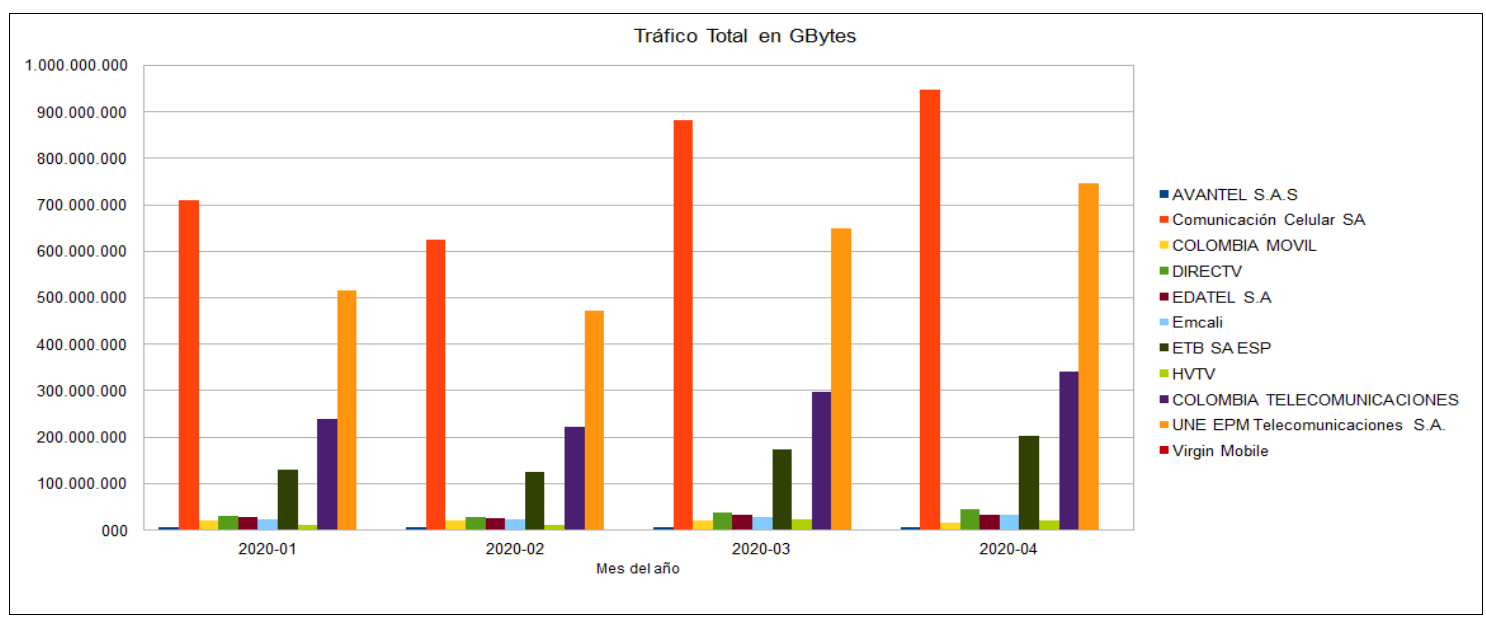

Fig. 1. Tráfico Total por operador en Colombia Enero a abril de 2020.

\section{B. Análisis del tráfico de Internet según la CRC}

Para hacer el análisis se tomaron los datos del informe de la CRC (Comisión de Regulación de las Comunicaciones) actualizado hasta el día 17 de Mayo [1]. Con base en estos datos se sacaron diferentes gráficos que muestran el comportamiento del tráfico de Internet en diferentes operadores durante la cuarentena por el Covid-19. A continuación se describirán los principales hallazgos encontrados a partir de dicho informe.

\section{1) Tráfico Total mensual enero-abril por operador}

Con base en los datos de la CRC se realizó inicialmente un gráfico del tráfico total en los meses de enero a abril de 2020 por cada operador (ver Fig. 1).

De la Fig. 1 podemos observar que:

- Los principales actores del internet en el país, es decir, los que se llevan el mayor ancho de banda son: Comunicación Celular SA, UNE EPM Telecomunicaciones SA, Colombia Telecomunicaciones, ETB SA ESP.

- Durante el mes de marzo en general se incrementó el tráfico de internet en los diferentes operadores. Es de anotar que desde el 20 de marzo se inició con un plan piloto de cuarentena en varias ciudades del país.

- El tráfico en general aumentó en todos los operadores durante el mes de abril, mes en el cual Colombia estuvo en Cuarentena total.

\section{2) Gráfico de Aumento de Tráfico en marzo y abril}

Ahora se hace un análisis de la proporción en que aumentó el tráfico para cada uno de los operadores de telecomunicaciones de Colombia en los meses de marzo y abril de 2020. Este aumento de tráfico se calculó como el tráfico en el mes respectivo dividido por el tráfico que hubo en febrero, el cual se tomó como línea base. Los resultados se observan en la Fig. 2.

En la Fig. 2 se observa que la mayoría de los operadores el aumento de tráfico en abril es mayor que el aumento en marzo. Adicionalmente vemos dos casos atípicos que son los de Colombia Movil y Virgin Mobile, los cuales disminuyeron su tráfico en abril, mientras que en marzo se mantuvieron casi igual que en febrero. Los demás operadores aumentaron su tráfico en marzo. En Abril EDATEL se mantuvo en el mismo valor de tráfico que en marzo y HVTV disminuyó el tráfico de abril respecto a marzo, claro está que como se observa HVTV había tenido un crecimiento del 100 por ciento (se duplicó) en el tráfico durante el mes de marzo y en abril fue uno de los más altos también a pesar de estar un poco más bajo que el mes anterior. Los demás tuvieron aumento de tráfico en abril respecto a marzo.

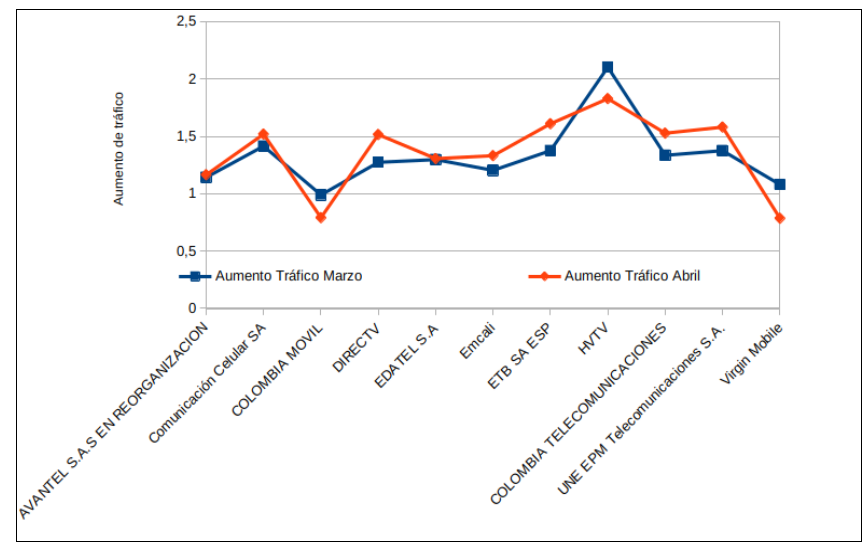

Fig. 2: Proporción de aumento del tráfico entre Marzo y Abril de 2020

A continuación, en la Fig. 3 se observa un gráfico de cajas de del comportamiento general de los operadores en cuanto al aumento de tráfico en marzo y abril. Se Puede observar que hubo tendencia general a aumentar el tráfico en mayor proporción en abril, aunque la varianza del tráfico de los operadores fue también mayor en ese mismo mes. La mediana del aumento de tráfico para marzo fue de 1.2976 (un 29.76\% de aumento) mientras que para abril fue de 1.5154 (un 51.54\% de aumento). Esto nos lleva a pensar que los operadores tuvieron que hacer grandes esfuerzos para incrementar su capacidad y evitar que llegasen a niveles de saturación sus redes y mantener una Calidad de Servicio aceptable para los clientes. 


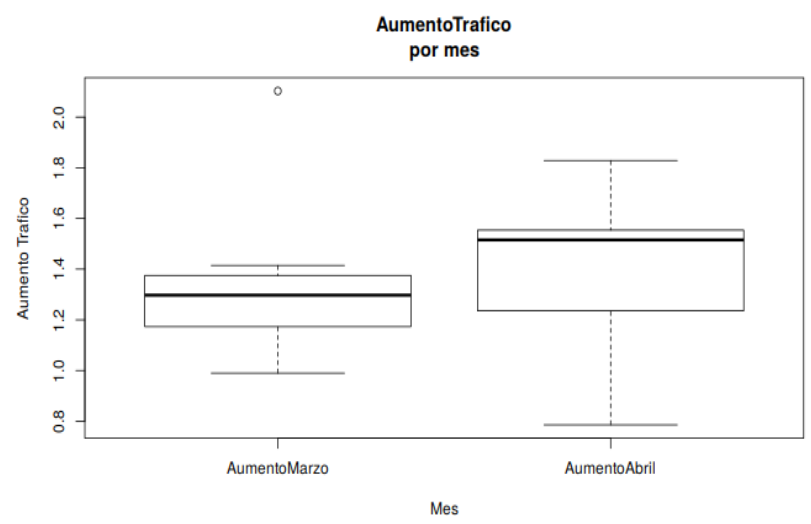

Fig. 3: Diagrama de cajas para la proporción de aumento del tráfico entre Marzo y Abril de 2020.

\section{3) Análisis del tráfico diario durante la cuarentena}

Otro análisis interesante es el comportamiento diario del tráfico durante la pandemia. Este se observa en la Fig. 4. En dicha figura se puede observar un patrón de comportamiento según el día de la semana, con una caída al iniciar la semana (lunes) y un aumento de tráfico los fines de semana (viernes, sábado y Domingo). Esto puede deberse al uso que se le da a Internet los fines de semana, el cual suele ser dedicado a actividades de esparcimiento y recreación tales como ver películas, reunirse virtualmente con amigos o jugar videojuegos.

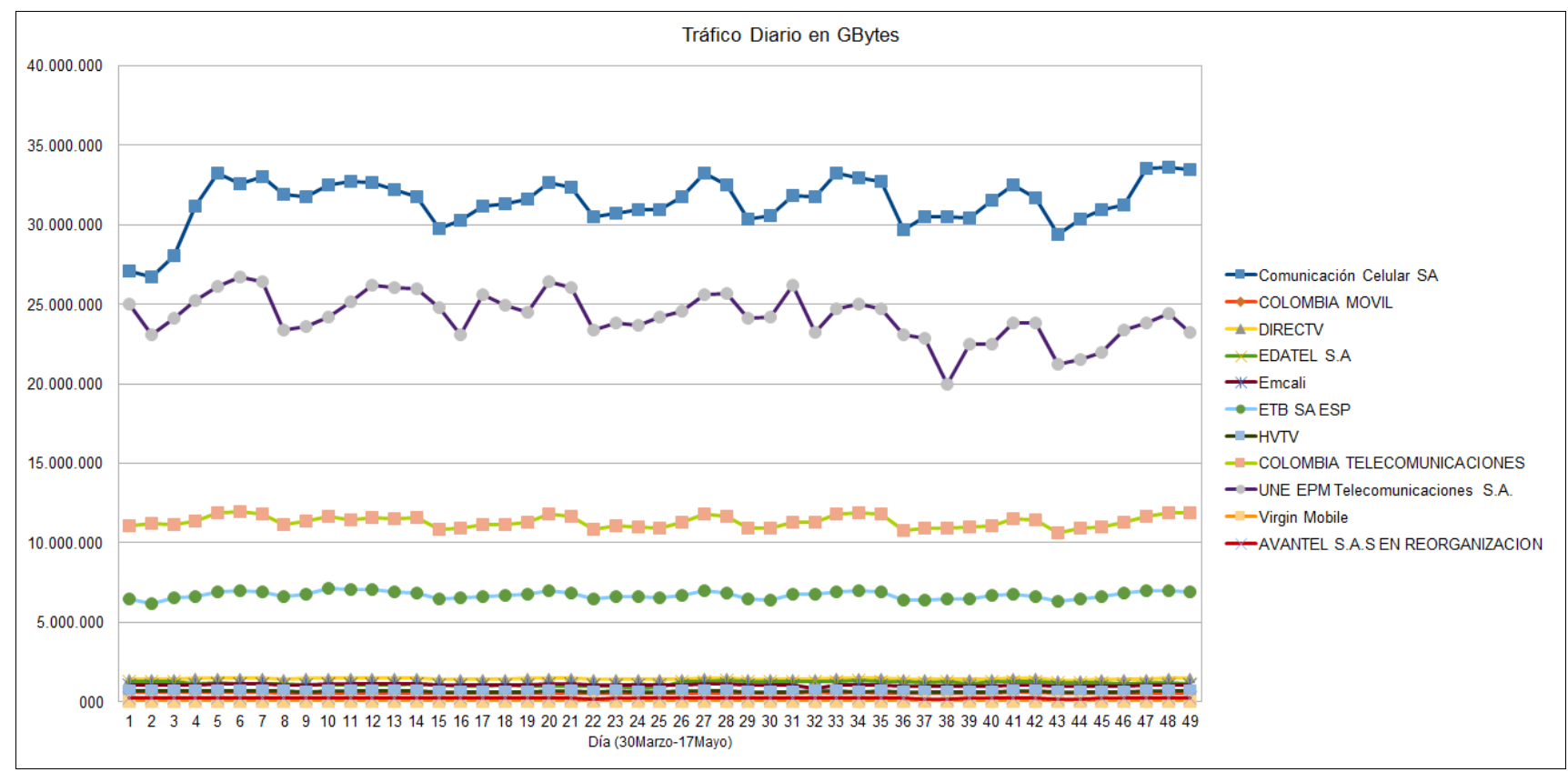

Fig. 4: Comportamiento del tráfico diario durante la cuarentena

\section{4) Análisis de la Hora Pico durante la Cuarentena}

Se graficó también el comportamiento de la hora pico durante la cuarentena para los diferentes operadores utilizando un histograma. Este se encuentra en la Fig. 5. En el histograma de la hora pico se observa que las horas de mayor tráfico estuvieron principalmente entre las 17 horas y las 22:59 horas. Los valores más frecuentes son las 18 horas y las 20 horas. Se esperaría que el mayor tráfico se presentara durante el día debido al Teletrabajo, pero no ocurrió así, tal vez esto se debe a que en las horas de la noche la gente suele acceder a servicios de películas, reunirse con amigos virtualmente o también a que los aficionados a los videojuegos suelen dedicarse a este entretenimiento en las horas de la noche.

\section{5) Análisis del tráfico Interno y externo por operador}

También se realizó un análisis del tráfico al interior de cada proveedor de Internet y el tráfico que intercambió con otros operadores. Para ello, se realizaron los gráficos de las Fig. 6 a Fig. 9.

Se observa en las Fig.6 a 9 que el tráfico en hora pico fue en aumento en los meses de marzo y abril, esto se debe a que a finales del mes de marzo ya se iniciaron los primeros intentos de hacer cuarentena mediante lo que se llamaron los Pilotos en Bogotá y en otras ciudades del país, mientras que todo el mes de abril estuvo ejecutándose la cuarentena completa a nivel Nacional. En general, en abril hubo un mayor tráfico en la hora pico. Este aumento se ve reflejado en el tráfico interno (tráfico local) y en el tráfico que se intercambia a nivel nacional (NAPs y Acuerdos de Peering) e Internacional. Se observa que el tráfico que se cruza entre operadores (NAP) es muy pequeño. También se observa que cada operador tiene sus conexiones Internacionales y que el tráfico Internacional es pequeño en comparación con el tráfico local. De los cuatro operadores, dos (Comunicación Celular SA y UNE EPM Telecomunicaciones SA) tienen acuerdos de tránsito o Peering 
directo y los otros dos (ETB y Colombia Telecomunicaciones) no tienen acuerdos de tránsito o no los usan.

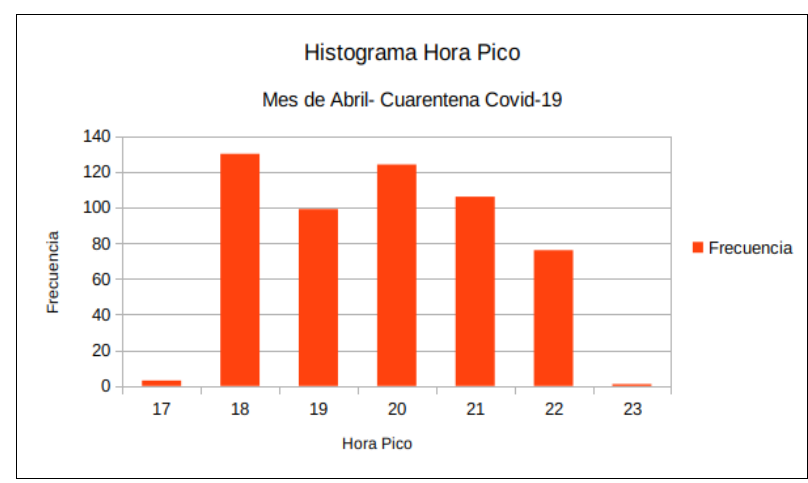

Fig. 5: Proporción de aumento del tráfico entre Marzo y Abril de 2020.

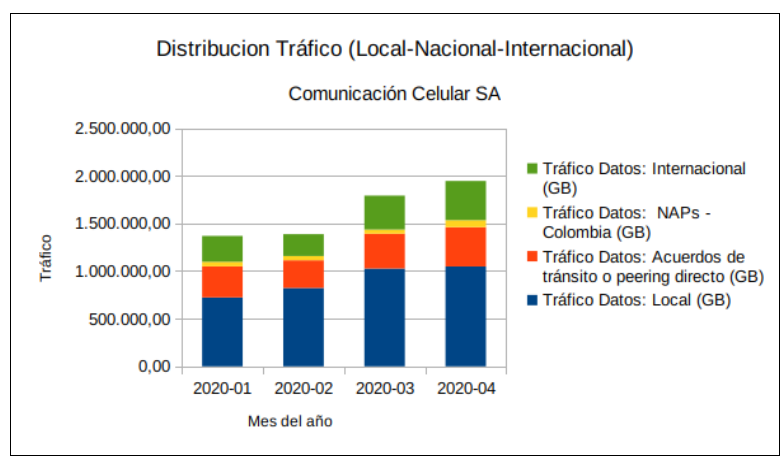

Fig. 6. Tráfico Interno y Externo en operador Comcel.

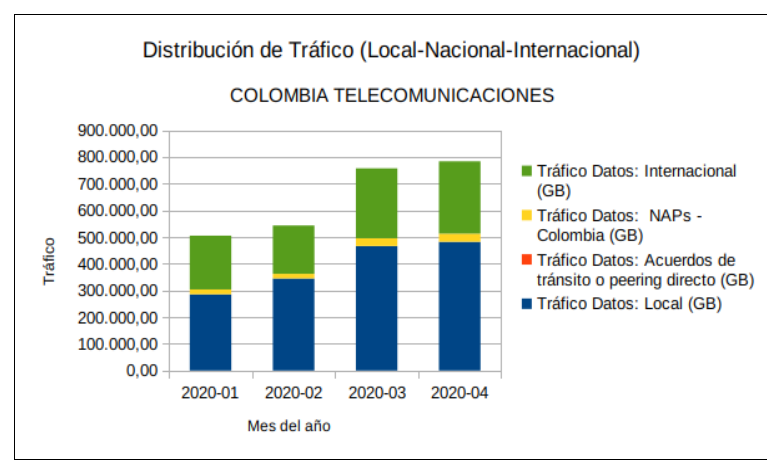

Fig. 7. Tráfico Interno y Externo en operador Movistar.

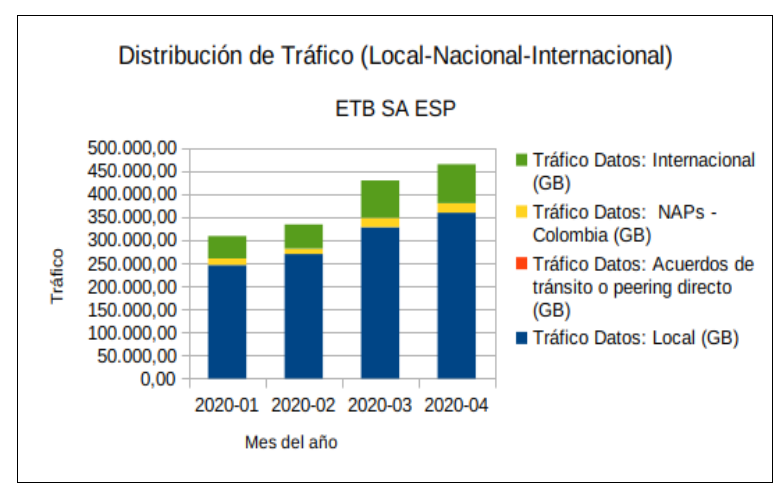

Fig. 8. Tráfico Interno y Externo en operador ETB.

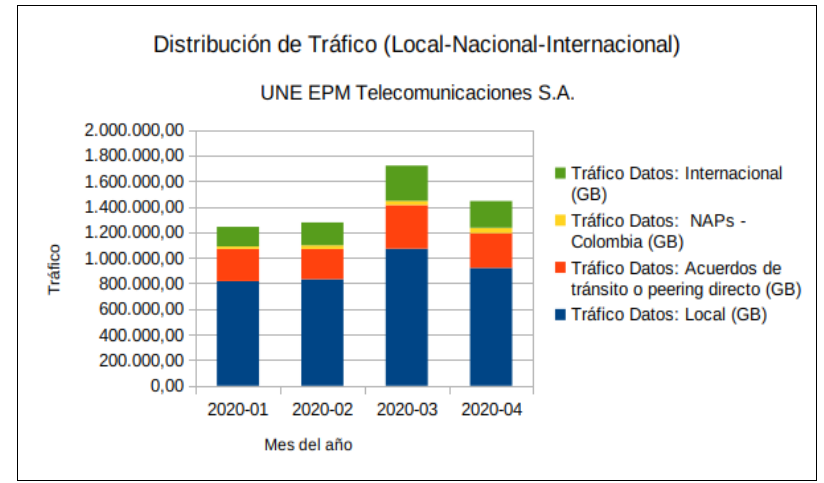

Fig. 9. Tráfico Interno y Externo en operador UNE.

\section{PROYECCIÓN DEL AUMENTO DE LOS RECURSOS EN LOS OPERADORES}

Una de las consideraciones que se debe tener es cuánto pudo haber costado al país este aumento del tráfico en Internet en términos de recursos, pues es claro que, al aumentar el tráfico, los recursos de los operadores debieron aumentar para mantener una Calidad de Servicio aceptable. Esta información sobre los recursos de cada operador no está disponible en el conjunto de datos entregado por la CRC, por lo que es necesario hacer una proyección de cuánto puede ser dicho aumento de recursos.

Para calcular este aumento de los recursos es necesario considerar un modelo de colas apropiado. Uno de los modelos más utilizado para el modelado de redes de datos es el modelo de Cola Cerrada o CQN o modelo de reparación de máquinas de Palm [6], que se puede observar en la Fig. 10.

En este modelo los nodos IS serían los terminales o clientes para atender y el nodo PS representa en nuestro caso una nube o internet que da servicio a las solicitudes de los clientes. Esta internet la modelamos de manera simplificada como un servidor y un buffer o cola de espera para los clientes. Los clientes en este caso generan tareas o solicitudes que van a ser procesadas por la nube y luego estas solicitudes son regresadas a los clientes. Este modelo puede utilizarse si se considera que hoy en día la mayor cantidad de tráfico utiliza el protocolo HTTP y que este protocolo a su vez utiliza el protocolo TCP para hacer control de la congestión extremo a extremo. Como es conocido, el modelo CQN es un sistema realimentado, que simula el efecto del algoritmo de control de la ventana de congestión de TCP conocido como arranque lento (slow start) [7]. A continuación, se describirá cómo opera el modelo CQN para luego describir cómo se utilizó para hacer las proyecciones.

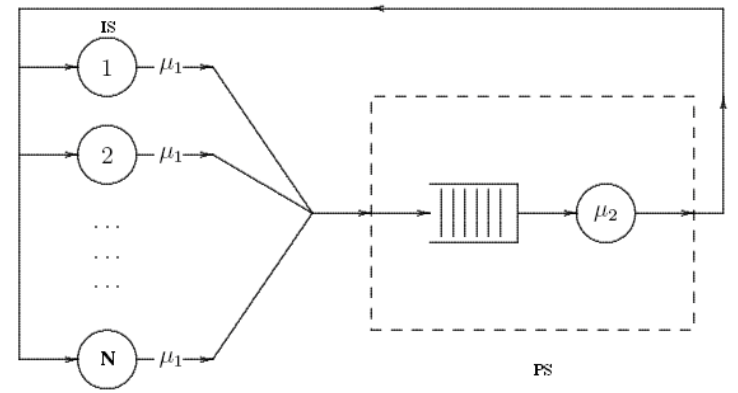

Fig. 10: Modelo de red de cola cerrada (CQN) [6] 


\section{A. Descripción del modelo CQN}

Para describir el modelo CQN o modelo de reparación de máquinas de Palm, utilizaremos la notación y descripción tal como se describe en [6]. Esta descripción es la siguiente:

$\mathrm{Se}$ consideran $\mathrm{S}$ terminales conectados a un Servidor. Se asume que los tiempos de "pensar" de cada terminal son largos. Se asumen entonces llegadas de Poisson exponencialmente distribuidas con intensidad:

$$
\gamma=1 / m_{t}
$$

El tiempo de servicio (ejecución) en el servidor se asume también exponencialmente distribuido con tasa:

$$
\mu=1 / m_{S}
$$

Para esta situación, el diagrama de estados se encuentra en la Fig. 11 y tiene las siguientes características:

- Para el diagrama de estados se asume el estado i que representa el número de terminales que comprende los terminales en cola y los terminales siendo atendidos.

- El Servidor está ocioso en: $i=0$.

- El Servidor está trabajando en: $i>0$, donde habrá $(i-1)$ terminales esperando.

- Se asume equilibrio estadístico.

- La intensidad de llegadas a la cola se decrementa en la medida que la longitud de la cola se incrementa (si todos los terminales están en cola, la intensidad de llegadas es cero).
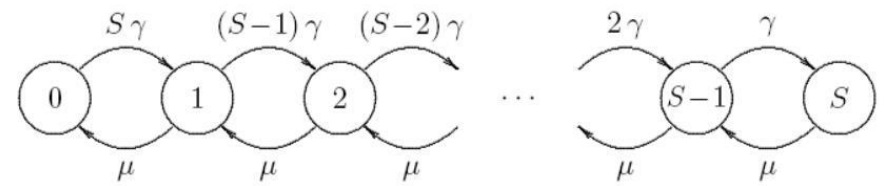

Fig. 11. Diagrama de estados de la red CQN [6]

Estableciendo las ecuaciones de corte o las de nodo se llega entonces a la expresión para $p(0)$, la cual es el estado en que todos los terminales están siendo atendidos o en espera de atención de su solicitud, es decir, $p(0)$ es la probabilidad de estar en la capacidad máxima del sistema. Por tanto, asumiendo que:

$$
A=\mu / \gamma
$$

y siguiendo el proceso de las ecuaciones de nodo o de corte se obtiene:

$$
\begin{gathered}
p(S-i)=\frac{A^{i}}{i !} p(S) \\
p(S-i)=\frac{\frac{A^{i}}{i !}}{\sum_{j=0}^{S} \frac{A^{I}}{j !}} \\
p(0)=E_{1, S}(A)
\end{gathered}
$$

Es decir, $p(0)$ tiene la forma de la distribución truncada de Poisson o expresión de Erlang B con $\mathrm{S}$ terminales o fuentes de tráfico. Por tanto, usando esta expresión se obtiene la relación entre el número de recursos (longitud de la cola necesaria para procesar S terminales), el grado de servicio y el tráfico ofrecido.

\section{B. Aplicación del modelo CQN}

Ahora se pasará a determinar la cantidad de tráfico que se inyectó a las redes de los diferentes operadores en los meses de febrero, marzo y abril de 2020. Esto se hace con el fin de calcular la cantidad de recursos adicionales que se requirieron en las diferentes redes utilizando la expresión de Erlang B que se demostró anteriormente era la apropiada para calcular la capacidad máxima del sistema modelado como una red de cola cerrada (CQN).

Para calcular la cantidad de tráfico tomaremos el modelo descrito en [8], el cual utiliza un modelo CQN para calcular el ancho de banda para tráfico elástico similar al producido por el protocolo HTTP. Este modelo utiliza una expresión basada en el tamaño medio de los archivos transferidos. La expresión obtenida y que fue probada en este artículo es:

$$
\mathrm{B}=\mathrm{N} * \mathrm{~h}
$$

Donde $\mathrm{B}$ es el ancho de banda total en la red, $\mathrm{N}$ es el número de fuentes de tráfico (usuarios o terminales), el cual es el mismo $\mathrm{S}$ de la fórmula para $\mathrm{p}(0)$ del modelo CQN, y h es la velocidad o ancho de banda por cada fuente de tráfico.

Por otro lado, en un estudio realizado en [10], se hizo un estudio del comportamiento de los usuarios en una red una red con tráfico predominante HTTP y se logró determinar con base en el análisis de las sesiones HTTP de diferentes muestras de tráfico tomadas en dicha red, que el valor de $h$ para ese estudio fue de $8900.78 \mathrm{bps}$, el cual tomaremos como un valor típico para nuestros cálculos. Cabe aclarar que esto es la velocidad generada para transmitir un objeto de una página web, lo cual se toma como una fuente de tráfico (usuario o tarea) a procesar en el modelo CQN

Por tanto, de la expresión 7 se puede despejar el número de fuentes de tráfico que se tienen como $\mathrm{N}=\mathrm{B} / \mathrm{h}$, donde $\mathrm{B}$ es el tráfico total ofrecido a la red, y que ya conocemos por las estadísticas entregadas por la CRC (Comisión de Regulación de Comunicaciones).

De otro lado, podemos calcular el tráfico en Erlangs generado por cada usuario como $\mathrm{a}=\mathrm{h} / \mathrm{r}$, donde $\mathrm{r}$ es la tasa de servicio en bits/segundo de la red. Asumimos que una red de estas tiene una velocidad de transmisión de $100 \mathrm{Gbps}$, lo cual es un valor actual típico para un enlace de fibra óptica del núcleo de la red. Por tanto, el valor del tráfico por cada fuente será de a $=8.90078 * 10-8$ Erl. Así, el tráfico total ofrecido a la red lo podemos calcular como $\mathrm{A}=\mathrm{a} * \mathrm{~N}$. Con esta expresión se obtuvieron los valores de tráfico ofrecido en Erlangs para los meses de febrero, marzo y abril para cada uno de los operadores. Luego, se hizo un cálculo del número de recursos necesarios para un Grado de Servicio de 0,02, el cual es un valor típico en una red de datos. El cálculo del número de recursos utiliza entonces la expresión de Erlang B, la cual ya fue demostrada que se usa como la probabilidad de llegar a la capacidad máxima del sistema. En esencia, el aumento en los recursos representa un aumento en el tamaño de las colas o buffers de la red CQN. Finalmente, se tiene la proporción de aumento de cada mes con respecto al mes de febrero, el cual 
se toma como la base para el cálculo, por lo que los meses de febrero tendrán una proporción de 1 .

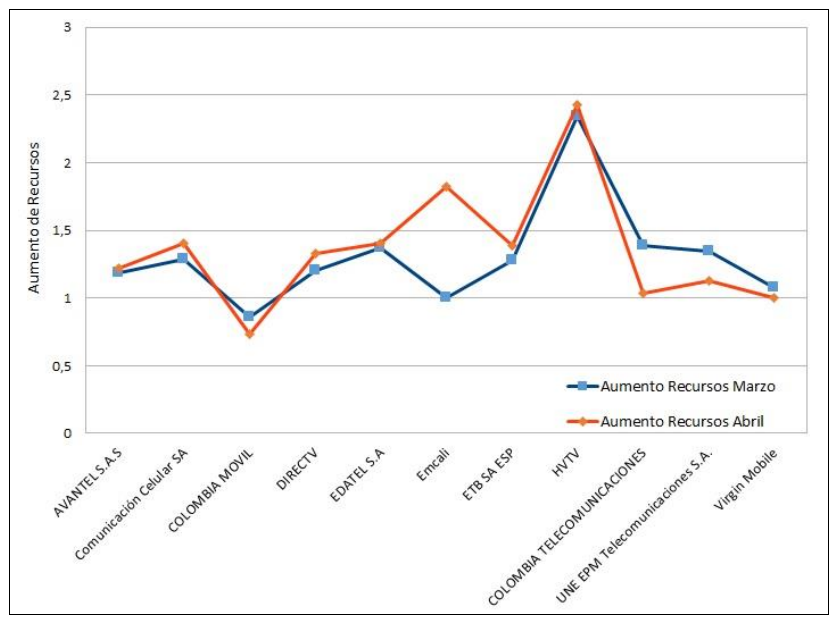

Fig. 12. Aumento de los Recursos calculado para los diferentes operadores.

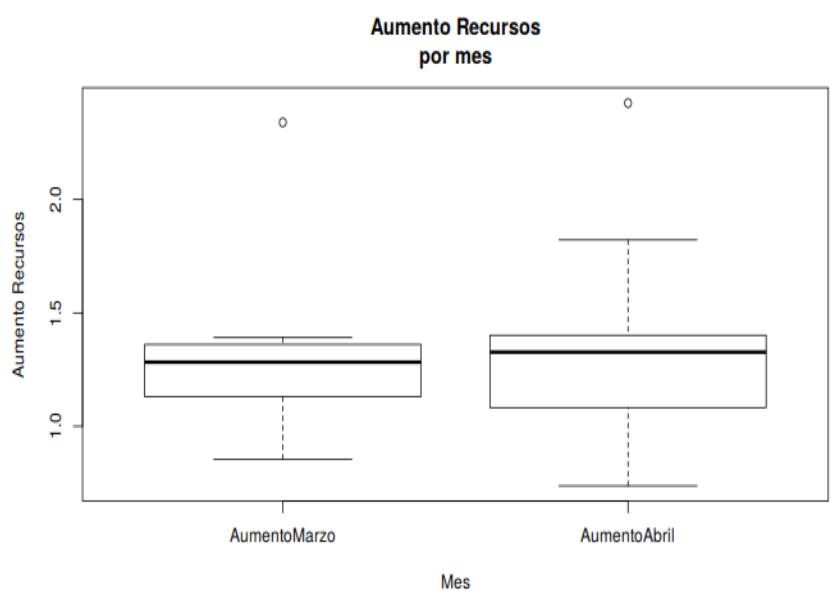

Fig. 13. Diagrama de caja para el Aumento de los Recursos para los diferentes operadores.

En la Fig. 12 se observa el comportamiento del aumento de recursos para los diferentes operadores con base en los resultados obtenidos con el modelo CQN. Adicionalmente, en la Fig. 13 se observa el diagrama de cajas para las proporciones de aumento en los recursos en las redes de los operadores. La mediana para el porcentaje de aumento de recursos en marzo fue de $28.3 \%$, mientras que para el mes de abril fue de $32.71 \%$. De otra parte, la varianza del aumento de recursos fue Mayor en abril pues el rango intercuartílico (IQR) es mayor para este mes. Por otro lado, el bigote superior del mes de abril llega más alto, lo que indica que hubo al menos un dato muy superior al cuartil 3, mientras que el bigote superior del mes de marzo es muy corto, mostrando que los datos más altos estuvieron muy cerca al tercer cuartil. Se observa que en ambas cajas hay un punto atípico superior al dos, que fue el caso de una red en particular con un aumento muy alto en el tráfico y que corresponde al operador HVTV.

\section{CONCLUSIONES}

En este artículo se analizaron los datos de tráfico en internet en Colombia suministrados por la CRC para los meses de enero a abril y hasta el 17 de mayo de 2020 con el fin de entender el efecto que tuvo la cuarentena por la pandemia del Covid-19 en las redes de Telecomunicaciones en Colombia.

Según el análisis realizado para los diferentes operadores, se observó en general un aumento en el tráfico de Internet del $29.76 \%$ para el mes de marzo, y un aumento del $51.54 \%$ para el mes de abril de 2020.

Además, se estableció un modelo para calcular el aumento de los recursos en las redes de los operadores de Internet para poder soportar este aumento de Tráfico. El modelo utilizado fue el de una cola CQN, el cual se utiliza ampliamente para modelar el efecto del tráfico del protocolo HTTP y el protocolo TCP con su algoritmo Slow Start usado durante la congestión. Para los cálculos se tomaron valores típicos de velocidades de transferencia de los objetos de una página $\mathrm{Web}$ mediante el protocolo HTTP.

Bajo estas condiciones se obtuvo una expresión basada en el modelo CQN descrito en [6] con el modelo de una red CQN para tráfico HTTP descrito en [8]. Como resultado se obtuvo el cálculo de los recursos adicionales necesarios para transportar el aumento de tráfico generado durante la pandemia del Covid-19. Los cálculos muestran que en general se debió aumentar la cantidad de recursos en un $28.3 \%$ en el mes de marzo y un $32.71 \%$ en el mes de abril de 2020 .

Con respecto a la hora pico del tráfico durante la cuarentena en marzo y abril, se encontró que los valores más frecuentes son las 18 horas y las 20 horas. Se esperaría que el mayor tráfico se presentara durante el día debido al Teletrabajo, pero no ocurrió así, tal vez esto se debe a que en las horas de la noche la gente suele acceder a servicios de películas, reunirse con amigos virtualmente o también a que los aficionados a los videojuegos suelen dedicarse a este entretenimiento en las horas de la noche.

En lo que tiene que ver con el análisis del tráfico interno y externo de las redes, en general, en abril hubo un mayor tráfico en la hora pico. Este aumento se ve reflejado en el tráfico interno (tráfico local) y en el tráfico que se intercambia a nivel nacional (NAPs y Acuerdos de Peering) e Internacional. Se observa que el tráfico que se cruza entre operadores (NAP) es muy pequeño. También se observa que cada operador tiene sus conexiones Internacionales y que el tráfico Internacional es pequeño en comparación con el tráfico local.

Por último, otro resultado que se obtuvo fue el análisis de la presión de Internet según los datos en [5] para las diferentes regiones de Colombia, obteniendo que el aumento de la presión de Internet tuvo un valor medio de $9.1 \%$, el cual es grande en comparación con otros países del primer mundo.

\section{REFERENCIAS}

[1] C. CRC, "CRC Colombia, Reporte del tráfico de Internet durante la Emergencia Sanitaria declarada por el Ministerio de Salud y Protección Social,"www.crcom.gov.co,2020.

https://www.crcom.gov.co/es/noticia/reporte-del-tr-fico-de-internetdurante-la-emergencia-sanitaria-declarada-por-el-ministerio-de-salud-yprotecci-n-social/.

[2] M. Favale, T. and Soro, F. and Trevisan, M. and Drago, I. and Mellia, "Campus traffic and e-Learning during COVID-19 pandemic," Comput. 
Networks, vol. 176, 2020.

[3] M. Branscombe, "The Network Impact of the Global COVID-19 Pandemic," www.thenewstack.io, 2020. https://thenewstack.io/thenetwork-impact-of-the-global-covid-19-pandemic/.

[4] J. Bergman, A. and Iyengar, "How COVID-19 is affecting internet performance," $\quad$ www.fastly.com, 2020. https://www.fastly.com/blog/how-covid-19-is-affecting-internetperformance/. plus $0.5 \mathrm{em}$ minus $0.4 \mathrm{em}$.

[5] D. Kaspr, "Slow Internet? How Covid-19 is Stressing Internet Infrastructure in Your Country," www.medium.com, 2020. https://medium.com/@kasprdata/slow-internet-how-covid-19-isstressing-internet-infrastructure-in-your-country-f94ee6e3b156/.

[6] V. Iversen, Teletraffic Engineering and Network Planning. Technical University of Denmark, 2006.

[7] N. A. L. Heyman, D.P., Lakshman, T.V., "A new method for analyzing feedback-based protocols with applications to engineering web traffic over the internet," ACM SIGCOMM Comput. Commun. Rev., vol. 23 , no. 4, 1993.

[8] Y. Berger, A. and Kogan, "Dimensioning Bandwidth for Elastic Traffic in High-Speed Data Networks," IEEE/ACM Trans. Netw., vol. 8, no. 5, 2000 .

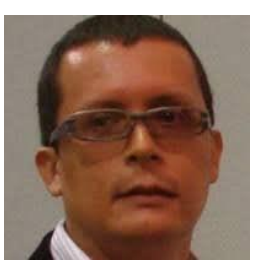

Jhon Jairo Padilla Aguilar. Doctor en Ingeniería Telemática por la Universidad Politécnica de Cataluña (2008). Magister en Informática por laq Universidad Industrial de Santander (1998). Ingeniero en Electrónica y Telecomunicaciones por la Universidad del Cauca (1993). Profesor Universitario desde hace 27 años. Es profesor universitario en la Universidad Pontificia Bolivariana en su sede de Bucaramanga y es el líder del grupo de investigación en informática y telecomunicaciones de la misma universidad. Ha sido coordinador de la Especialización en Telecomunicaciones y de la Maestría en Ingeniería Electrónica de la UPB. Ha impartido cursos en diferentes instituciones nacionales e Internacionales como la ITU y la Universidad Nacional de Asunción. Ha hecho parte de la red de investigadores RIEGONETS sobre el uso de la electrónica y las Telecomunicaciones en la agricultura. Sus áreas de interés son: Redes Inalámbricas, Internet, IPv6, Teletráfico, Ingeniería de tráfico en Internet y Data Mining. ORCID: https://orcid.org/0000-0002-8552-2873. 